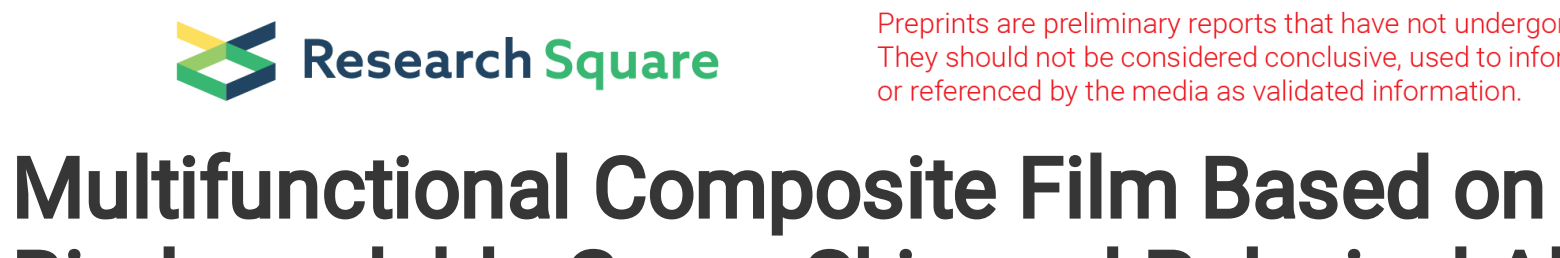 \\ Biodegradable Grape Skin and Polyvinyl Alcohol
}

\section{Zhe Qiu}

Northeast Forestry University

Weidong Niu

Northeast Forestry University

Shuo Wang

Northeast Forestry University

Fanjun Yu

Northeast Forestry University

Yang Yu

Northeast Forestry University

Jing Fan

Northeast Forestry University

Longhong Zheng

Northeast Forestry University

Yonggui Wang ( $\nabla$ wangyg0617@163.com )

Northeast Forestry University https://orcid.org/0000-0002-5135-1367

Zefang Xiao

Northeast Forestry University

Yanjun Xie

Northeast Forestry University

\section{Research Article}

Keywords: grape skin, cellulose nanofiber, PVA, polyphenols, multifunctional, composite film

Posted Date: February 11th, 2021

DOI: https://doi.org/10.21203/rs.3.rs-184116/v1

License: (9) (1) This work is licensed under a Creative Commons Attribution 4.0 International License.

Read Full License 


\section{Abstract}

In winemaking, large amounts of grape skin (GS) are produced as by-products, which contain not only abundant degradable cellulose, hemicellulose, and pectin but various functional polyphenols. In contrast to most studies focusing on the utilization of extractives, the current study investigates the use of an ultrasonicated grape skin (UGS) containing all components to develop a multifunctional composite film. Owing to dissociation during ultrasonication, all GS components were well dispersed in water to obtain the UGS suspension. Transmission electron microscopy (TEM) indicated that the celluloses were successfully transformed into cellulose nanofibers (CNFs), which can improve the uniformity of the composite film. Subsequently, biodegradable and multifunctional composite films were fabricated by combining the UGS and polyvinyl alcohol (PVA). The UGS and PVA formed a good interface owing to strong hydrogen bonds, and the resulting films exhibited excellent thermal stability and moisture-sensitive mechanical properties. The polyphenols in the UGS suspension endowed the composite film with multiple functions, including $\mathrm{pH}$-responsive color change, excellent antioxidant activity, ultraviolet shielding, and antimicrobial properties. The use of PVA enhanced the flexibility, strength, and elongation of the UGS film. The easily prepared, tailored, multifunctional, and biodegradable UGS/PVA composite film exhibits excellent potential for application in agriculture, cosmetics, and healthcare.

\section{Introduction}

Grape wine is a widely known drink, and the grape industry has a large market share. More than 60 million tons of grapes are harvested annually, 75\% of which is used in winemaking (Fig. 1a), resulting in $~ 14.5$ million tons of grape pomace by-product (Beres et al. 2017; Ferrari et al. 2019; Fan et al. 2020). Grape skin (GS) is the major component of grape pomace, comprising roughly half of the material weight (Mendes et al. 2013). GS is currently not considered a highly profitable waste and is discarded, causing environmental problems. Only a small proportion of GS is used for animal feed. The lack of sufficient applications for GS, as well as the increasing environmental concern, has drawn widespread interest. The GS, which is similar to a porous hydrogel, is presented in Fig. $1 \mathrm{~b}$. From the outside to the inside, the porous skeleton of GS consists of the cuticle, intermediate epidermis, and hypodermis (Fig. S1) (Ortega-Regules et al. 2008). Dried GS consists of cellulose, hemicelluloses, polyphenols, pectin, proteins, lipids, and soluble sugar (Mendes et al. 2013). The hypodermis contains most of the polyphenols in GS (Lecas and Brillouet 1994). Although cellulose represents the largest proportion of GS, most studies have focused on the extraction and use of various extractives, such as anthocyanins, hydroxycinnamic acids, flavanols, and flavonol glycosides (de Moura et al. 2002; EsquivelAlvarado et al. 2020; Lavelli et al. 2016; Quijada-Morín et al. 2015; Xu et al. 2018; Zhu et al. 2015). These extractives represent only a small proportion of the GS, and a significant portion is wasted. Considerable progress effort is required to achieve the comprehensive utilization of the whole GS.

The increasing environmental concern has resulted in the strong inclination to use so-called "green materials" to replace petroleum-based non-degradable plastic films. Biodegradable plant-based materials (cellulose, starch, and polylactic acid) are desirable alternatives to petroleum-based products because 
they are environmentally friendly (Liu et al. 2020; Wang et al. 2020; Yu et al. 2020). However, as market demand rises, the overuse of plant-based films may create a new ecological and food crisis (Han et al. 2018; Papadopoulou et al. 2019; Sun et al. 2019; Tu et al. 2020; Wang et al. 2018). Large amounts of plant cellulose (fruit skins, stems, crop husk, leaves, and corncobs) are wasted as by-products in the food industry. Owing to their high biodegradability, most by-products end up in landfills. Cellulose resources are significantly wasted, and the polyphenols in the by-products may cause groundwater pollution (Jin et al. 2020). Therefore, the efficient use of cellulose in food by-products not only minimizes the imbalance between the supply and demand of the biodegradable materials but also reduces resource wastage and environmental pollution. As one of the aforementioned by-products of winemaking, GS shows significant potential because of its high cellulose content. Naturally dried GS is brittle because of its high pectin content and deficiency in mechanical properties required for creating a film (López de Lerma et al. 2014; Šešlija et al. 2018). Thus, the combination of GS with a suitable material represents a good solution. Poly(vinyl alcohol) (PVA) is a water-soluble and biocompatible polymer that has been studied extensively owing to its excellent film-forming properties and complete biodegradability (Liu et al. 2018). Their excellent interfacial compatibility and the simplicity of the preparation have prompted the research and development of many cellulose/PVA composite films. These films exhibit satisfactory mechanical strength and flexibility (Kim et al. 2019; Yang et al. 2020; Xu et al. 2018). Moreover, cellulose and PVA can be combined in an aqueous solution, which is beneficial for maintaining the activities of functional polyphenols in GS.

In the present study, a biodegradable functional composite film was successfully fabricated by mixing an ultrasonicated GS (UGS) suspension with PVA (Fig. 1C). The cellulose nanofibers (CNFs) formed a threedimensional (3D) porous skeleton to support the composite film. The hemicellulose and pectin in the plant cell wall acted as the mechanical support and adhesive substance (Kozioł et al. 2017), ensuring the formation of the film and improving the interface bonding between the UGS and PVA. The self-standing UGS-PVA composite film was formed after drying the mixed solution at $35^{\circ} \mathrm{C}$ for $12 \mathrm{~h}$. Moreover, the polyphenols endowed the film with desirable multiple functions, including $\mathrm{pH}$-responsive color change, antioxidant activity, ultraviolet (UV) shielding, and antimicrobial properties. The multifunctional biodegradable composite film has considerable potential in cosmetics, health care, and agriculture.

\section{Materials And Methods}

\section{Materials}

GS was obtained from fresh grapes (Vitisvinifera $c v$. Kyoho). PVA (AR $>97 \%$ ) was supplied by the Tianjin Guangfu Fine Chemical Research Institute (Tianjin, China). 1,1-diphenyl-2-picrylhydrazyl (DPPH) and sodium hydroxide $(\mathrm{NaOH})$ were purchased from Shanghai Macklin Biochemical Technology Co., Ltd. (Shanghai, China). Ethanol $\left(\mathrm{C}_{2} \mathrm{H}_{5} \mathrm{OH}\right)$ and methanol $\left(\mathrm{CH}_{3} \mathrm{OH}\right)$ were provided by Shanghai Aladdin Biochemical Technology Co., Ltd. (Shanghai, China). Hydrochloric acid $(\mathrm{HCl})$, magnesium nitrate 
$\left(\mathrm{Mg}\left(\mathrm{NO}_{3}\right)_{2}\right)$, and potassium nitrate $\left(\mathrm{KNO}_{3}\right)$ were purchased from Damao Chemical Reagent Factory (Tianjin, China).

\section{Preparation of the GS}

The fresh GS was washed with distilled water until no stainswere observed on the outside surface of the skin, and no grape pulp adhered to the inside surface of the skin. The washed GS was then freeze-dried (Scientz-30ND, Ningbo Scientz Biotechnology Co., Ltd., Ningbo, China) to obtain oven-dried GS. To prevent the growth of yeast and mildew, $75 \% \mathrm{C}_{2} \mathrm{H}_{5} \mathrm{OH}$ was sprayed onto the dry GS. The sterilized GS was again dried in a vacuum drying oven (DZ-1AZ, Tianjin City Taisite Instrument Co., Ltd., Tianjin, China) at $35^{\circ} \mathrm{C}$ for $12 \mathrm{~h}$ and then stored in a desiccator.

\section{Preparation of the UGS suspension}

The GS was ground into powder using a pulverizer (BO-2500Y, BoouHardware Products Co., Ltd., Zhejiang, China) and sieved through a 600 mesh screen to prepare the UGS suspension. The GS powder was mixed with distilled water (1\% wt) and stirred for $15 \mathrm{~min}$ at $800 \mathrm{rpm}$. The obtained GS powder suspension was treated with an ultrasonic cell disruptor (JY 99-凶D, Ningbo ScientzBiotechnology Co., Ltd., Ningbo, China) at $800 \mathrm{~W}$ for $4 \mathrm{~h}$ to dissociate the cell walls and obtain aninitial UGS suspension. The initial UGS suspension was then centrifuged (H1850, Hunan XiangYi Laboratory Instrument Development Co., Ltd., Changsha, China) at $150 \mathrm{rpm}$ for $10 \mathrm{~min}$ to remove the large segments. The obtained UGS suspension was adjusted to $0.1 \% \mathrm{wt}$. for further use.

\section{Preparation of the UGS-PVA film}

PVA and distilled water were added to abeakerat a ratio of 1:9 (wt). The sealed beaker was placed in a water bath and then heated $\left(95^{\circ} \mathrm{C}\right)$ and stirred $(200 \mathrm{rpm})$ until the PVA was completely dissolved. The PVA solution was then cooled to room temperature. UGS-PVA mixed solutions were prepared with solid UGS: PVA concentration ratios of 0:100, 5:95, 10:90, and 15:85. The mixed solutions were placed in superflat plastic Petri dishes and dried at $35^{\circ} \mathrm{C}$ for $12 \mathrm{~h}$. The obtained composite films were assigned as PVA, UGS ${ }_{5}-$ PVA, $U_{G S} S_{10}-P V A$, and $U_{G S}{ }_{15}-P V A$.

\section{Characterization}

The chemical structure of the UGS was characterized using a Fourier transform infrared (FTIR) spectrometer (ZN-04, KINGSLH, China) and a solid-state ${ }^{13} \mathrm{C}$ NMR spectrometer (AVANCE III $400 \mathrm{MHz}$ WB, Bruker, Switzerland). The surface morphology of the films was characterized using an atomic force microscope (AFM, Dimension Icon, Bruker, Germany). Transmission electron microscopy (TEM) was 
performed on a Tecnai G20 electron microscope (JEM 2100) with an acceleration voltage of $200 \mathrm{kV}$. Scanning electron microscopy (SEM, JSM-7500F, JEOL, Japan) was conducted to observe the morphology of the films. The crystalline structure of the films was characterized using anoptical polarizing microscope (CX40P, Ningbo, China). The degree of crystallinity index of the films was determined using an X-ray diffractometer (XRD, D8 Advance, Bruker, Germany.) The thermal stability of the film was analyzed with a thermogravimetric analyzer (TGA/DSC 1/1600 HF Mettler-Toledo, Switzerland) at a heating rate of $10^{\circ} \mathrm{Cmin}^{-1}$ in a nitrogen environment (from room temperature to $700^{\circ} \mathrm{C}$ ). The optical properties of the films were analyzed using a UV-visible (UV-vis) spectrophotometer (TU-1950, Beijing Purkinje General Instrument Co., Ltd., Beijing, China). A testing machine (Al-7000S TC160701511, Gotech, Taiwan) was used to evaluate the mechanical properties of the films.

\section{Moisture adsorption test}

Two sealed glass containers with internal relative humidity $(\mathrm{RH})$ levels of $56 \%$ and $93 \%$ were prepared using the saturated salt solution method with $\left(\mathrm{Mg}\left(\mathrm{NO}_{3}\right)_{2}\right.$ and $\mathrm{KNO}_{3}$ at a constant temperature of $20^{\circ} \mathrm{C}$. Dried PVA and UGS-PVA films measuring $1 \mathrm{~mm} \times 1 \mathrm{~mm}$ were placed in the containers until the film weight increased by $<0.005 \mathrm{~g}$ within $24 \mathrm{~h}$ at a given $\mathrm{RH}$. The moisture adsorption of the film was based on moisture content (MC), which was determined using Eq. 1:

\section{$M C(\%)=\left(m_{1}-m_{0}\right) / m_{0} \times 100 \% \quad(1)$,}

where $m_{0}$ is the dry weight of the films, $m_{1}$ is the equilibrium weight at a given $R H$, and $M C$ is equal to the equilibrium MC. Eight replicates of each film were used for each RH.

\section{Antioxidant activity test}

With the UV-vis spectrophotometer, the antioxidant activity of the UGS-PVA films was measured based on the disappearance of the absorption band at $517 \mathrm{~nm}$ of the DPPH free radical. A $0.1 \mathrm{~mol} / \mathrm{L}$ test solution was prepared by dissolving DPPH in $\mathrm{CH}_{3} \mathrm{OH}$, and the solution was covered with foil to shield it from light. Film-soaking solutions were prepared by soaking $0.15 \mathrm{~g}$ of the film in $3 \mathrm{~mL}$ of $\mathrm{CH}_{3} \mathrm{OH}$ for 30 min. Subsequently, $2 \mathrm{~mL}$ of the DPPH solution and $2 \mathrm{~mL}$ of the film soaking solution were added and mixed in glass vials wrapped in foil for $30 \mathrm{~min}$. The mixed solution was ultimately analyzed using the UVvis spectrophotometer. The radical scavenging activity (RSA) of the films was calculated using Eq. 2:

\section{$\operatorname{RSA}(\%)=\left(A_{c t r}-A_{\text {sample }}\right) / A_{c t r} \times 100 \% \quad(2)$,}

where $A_{c t r}$ and $A_{\text {sample }}$ are the absorbance values of the control and the films at a wavelength of $517 \mathrm{~nm}$, respectively. 


\section{$\mathrm{pH}$ responsivenesstest}

The level of $\mathrm{pH}$ responsiveness was measured using a portable colorimeter (CM-2300D, MINOLTA) equipped with a CIELAB system. Aqueous solutions with different $\mathrm{pH}$ values $(1,3,5,7,9,11$, and 13$)$ were prepared usingdistilled water, $0.1 \mathrm{~mol} / \mathrm{L} \mathrm{HCl}$, and $0.1 \mathrm{~mol} / \mathrm{L} \mathrm{NaOH}$. The solutions were dropped onto the UGS $_{15}$-PVA film measuring $1 \mathrm{~cm} \times 1 \mathrm{~cm}$, respectively. After sealing and resting for $30 \mathrm{~min}$, the color of the films was determined.

\section{Antimicrobial simulation test}

Two plastic Petri dishes $6 \mathrm{~cm}$ in diameter were sterilized by UV light, and $20 \mathrm{~mL}$ of milk was placed into both Petri dishes. The PVA and UGS ${ }_{15}$-PVA film measuring $2 \mathrm{~cm} \times 2 \mathrm{~cm}$ were placed into the two Petri dishes. Both Petri dishes were sealed and stored for 3 days to ensure the migration of polyphenols in the film into the milk. The seal was subsequently removed, and the dishes wereplaced in the laboratory for about 3 weeks. The final state of the milk was determined using photographs.

\section{Results And Discussion}

\section{Preparation of UGS and fabrication of the UGS-PVA films}

The dried GS with a porous skeleton was first pulverized into powder measuring 10-20 $\mu \mathrm{m}$ (Fig. 2a-2c), which further underwent ultrasonication treatment for sufficient dissociation and good dispersion of the UGS suspension. The ultrasonic impact disintegrated the grape cellulose into the CNF with a high specific surface area and a diameter of 4-10 nm, resulting in the sufficient explosion of large amounts of the hydroxyl group (Fig. 2d). Thus, the CNF and aqueous solution formed a uniform and stable 3D network attributable to hydrogen bonds (Chen et al. 2011). In addition, the TEM image showed the presence of aggregates, which formed as core-shell structures measuring $\sim 100 \mathrm{~nm}$ (Fig. 2d-2e). Consisting of proteins or lipids, the core was encapsulated by polyphenols as shells due to hydrogen bonds (Liu et al. 2019a; Zhang et al. 2020).

In addition to efficient dissociation of cellulose into CNFs, the polyphenols, pectin, proteins with multiple functions were successfully maintained after ultrasonication, as determined by FTIR and NMR spectroscopy (Fig. 2f-2g). As shown in Fig. 2f, the UGS filtrate has adsorption peaks at 2918 and 2849 $\mathrm{cm}^{-1}(\mathbb{\nabla})$ which are ascribed to the asymmetric and symmetric stretching vibration of $\mathrm{CH}_{2}$ in the lipids. The peaks at 1732 and $1716 \mathrm{~cm}^{-1}$ (ii) were attributed to the $\mathrm{C}=0$ stretching vibration of the aliphatic

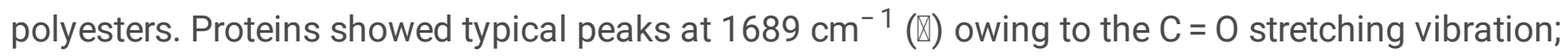
however, the peak was weak because the protein content of the GS was less than 5\% (Pinelo et al. 2006). The peak at $1605 \mathrm{~cm}^{-1}(\mathbb{D})$ was ascribed to the $C=0$ asymmetric stretching vibration of the pectin and $C$ $=\mathrm{C}$ stretching vibration of the polyphenols. The pectin had adsorption peaks at vi, vii, and viii attributed to the $\mathrm{CH}_{2}, \mathrm{OH}, \mathrm{C}-\mathrm{O}$, and $\mathrm{C}-\mathrm{C}$ stretching vibrations, respectively. The peaks at $\otimes$ were ascribed to $\mathrm{C}-\mathrm{H}$ 
deformation in the polyphenols. The FTIR peaks are described in detail in Table S1. The polyphenols were analyzed by solid-sate ${ }^{13} \mathrm{C}$ NMR. As shown in Fig. $2 \mathrm{~g}$, the filtered UGS primarily consists of pectins (170$182 ; 105.2 ; 53.6 \mathrm{ppm})$, holocelluloses (62-106 ppm), residual polyphenols (115-155 ppm), and lipids (30 ppm) (Castillo-Muñoz et al. 2009; Farooque et al. 2018; Matharu et al. 2016; Mendes et al. 2013; Zhu et al. 2014). The dominant polyphenols included tannins, anthocyanins, proanthocyanidins, andflavonols, which exhibited typical peaks at 55-155 ppm (Mateus et al. 2002; Prozil et al. 2012). The UGS filtrate also contained pectins, proteins, and lipids, those substances combined with the polyphenols and formed functional aggregates (Fig. 2e). Details of the NMR results are listed in Table S2.

Transparent and self-standing brown UGS-PVA films were fabricated by solvent casting of the mixtures of UGS and PVA solution (Fig. 2a). After the water evaporated, transparent films with homogenous distribution of UGS were obtained (Fig. 2a). The surface roughness increased slightly relative to that of the pure PVA film (Fig. 3a, 3b, and S2). The homogeneous distribution of UGS in the composite films is ascribed to sufficient dissociation by ultrasonication and the presence of numerous hydroxyl groups, which can strongly interact with PVA chains via hydrogen bond formation. This occurrence led to superior compatibility between PVA and UGS at the interface and prevented the debonding of the UGS substrate from the matrix, as observed in the SEM images (Fig. $3 \mathrm{~b}_{2}$ ). By contrast, GS without ultrasonication, which contained micro-scaled particles, could not feasibly be used to fabricate such homogenous films but instead, only films with considerably aggregated GS particles (Fig. S3). Thus, the nano-dimension of UGS by ultrasonication is critical for the formation of homogenous transparent and self-standing composite films. In addition, solely drying UGS failed to generate stable self-standing films and only resulted in small cracks (Fig. S4).

The addition of UGS effectively influenced the crystal nucleation of PVA. As shown in Fig. $3 a_{1}$ and $3 b_{1}$, the morphological surface of pure PVA is relatively smooth. This smoothness can be attributed to the low crystallization of pure PVA chains, which renders most PVA molecular chains amorphous during water evaporation. However, the surface of the UGS-PVA composite film was uneven and rough, with numerous grains and gullies (Fig. $3 \mathrm{~b}_{1}$ ). These qualities could be caused by the improvement of crystallization after the addition of UGS; some PVA chains crystallized into ordered domains, whereas the others were amorphous. This occurrence was verified by the significant increase in the crystallinity index $\left(C_{r}\right)$ as UGS was added (Fig. 3c). Pure PVA had a $C_{r} /$ of $26.82 \%$, and $\mathrm{UGS}_{15}-\mathrm{PVA}$ had a $C_{r} /$ of $61.26 \%$. On the basis of the polarized light microscopy measurement, well-distributed bright domains were visible in the composite film, with a higher density than that of the pure PVA film, indicating significant improvement in the crystallization of the UGS-PVA composite film. Moreover, the presence of typical peaks ascribed to UGS within the FTIR spectra confirmed the successful maintenance of all UGS components in the composite film, particularly polyphenols, which are critical for the introduction of new functions in the composite films (Fig. 3d). The UGS-PVA composite films exhibited thermal stability superior to that of the pure PVA film because the cellulose and pectin showed higher thermal stability than the PVA (Einhorn-Stoll et al. 2007; Kaboorani et al. 2012) (Fig. 3e). Specifically, with an increase in UGS content from $0-15 \%$, the first thermal decomposition peak of the film shifted from $249^{\circ} \mathrm{C}$ to $289^{\circ} \mathrm{C}$. 
This improvement in thermal stability would be beneficial for the practical application of the UGS-PVA composite films.

\section{Moisture-sensitive mechanical properties of the UGS-PVA films}

As shown in Fig. 4a, the dried UGS ${ }_{5}$-PVA film with excellent flexibility and toughness can be easily folded into an airplane; no damage occurs after the film is unfolded. Similar to the pure PVA film, the composite films exhibited moisture-switchable mechanical properties. In its wet state, the UGS ${ }_{5}-P V A$ film could attach to the finger and be easily deformed without damage when the finger was bent, exhibiting excellent softness and adhesion (Fig. 4b). Moreover, the wet $\mathrm{UGS}_{5}-\mathrm{PVA}$ film could be stretched to about twice its original length and was restored to its original shape (Fig. 4c), revealing an elastic mechanical behavior after stretching. To understand the influence of moisture on the mechanical properties, moisturedependent analyses were further performed. As shown in Fig. 4d, the MCs of all films increase as RH rises from 56-93\%. The pure PVA film with a semi-crystalline structure could be easily destroyed by moisture, resulting in efficient moisture adsorption at increased RH (Konidari et al. 2011). Unlike that in PVA film and UGS-PVA films with similar MC levels at low RH (56\%), the addition of UGS impeded moisture adsorption at a high RH level (93\%) because of the enhancement of the crystalline structure (Fig. 3c), leading to lower MC of the UGS-PVA film than that of the PVA film (Fig. 4d). In Fig. 4e, the crystalline domains in the $\mathrm{UGS}_{15}-\mathrm{PVA}$ film almost disappears after moisture equilibrium treatment at high $\mathrm{RH}$ and vice versa after drying. This occurrence endowed the composite film with moisture-sensitive mechanical properties.

The tensile curves of all films at different RH levels are presented in Fig. $4 \mathrm{f}-4 \mathrm{~g}$. In its dry state, the pure PVA film exhibits the highest strength of $95.3 \mathrm{MPa}$, with a breaking elongation of $5.6 \%$. For the UGS-PVA films, the strength decreased from $76.6 \mathrm{MPa}$ to $13 \mathrm{MPa}$, and the breaking elongation was reduced from $3.2-1.2 \%$ as the UGS content increased from $5-15 \%$. After the films were equilibrated at RH levels of $56 \%$ and $93 \%$, their strengths decreased, and their breaking elongations increased significantly (Fig. $4 \mathrm{~g}-4 \mathrm{~h}$ and Fig. S6). The UGS 15 PVA film treated at RH of $93 \%$ exhibited its lowest strength of $12.1 \mathrm{MPa}$ and breaking elongation of $412.3 \%$, which still satisfied the standard requirement for polyethylene film in packaging applications ((strength $\geq 10.5 \mathrm{MPa}$ and breaking elongation $\geq 100 \%$ ).(2016) The significant increase in breaking elongation at a high $\mathrm{MC}$ is attributed to the dissociation of the crystalline structure, which would facilitate the application of UGS-PVA films in packaging, wound dressing, and cosmetics, among others.

\section{Multiple functions of UGS-PVA films}

The polyphenols in the UGS endowed the UGS-PVA films with multiple functions. As shown in Fig. 5a, the UGS ${ }_{15}$-PVA film exhibits $\mathrm{pH}$-induced color switch ability. The color could change from soft pink to 
blue/brown with an increase in $\mathrm{pH}$ from 1 to 13 . This change is ascribed to the chemical structural change in anthocyanin in the GS (Fig. S7). The anthocyanin gradually became deprotonated as the pH changed from acidic to basic (Fig. S8). Red flavylium cations $\left(\mathrm{AH}^{+}\right)$formed and became predominant at $\mathrm{pH} 1-3$. When the $\mathrm{pH}$ increased to 4-6, a colorless hemiketal formed, and yellow chalcone developed via ringopening. The $\mathrm{AH}^{+}$was ultimately transformed into purple-blue quinonoid base isomers $(\mathrm{pH}>6)(\mathrm{Liu}$ et al. 2019b; Sigurdson et al. 2017). Moreover, the anthocyanin also exhibits considerably high antioxidant activity (Serra et al. 2008; Št'avíková et al. 2011). The combination of anthocyanin with other GS antioxidants, such as proanthocyanidins and resveratrol, endowed the UGS-PVA films with high antioxidant activity (Fig. 5b-5c). DPPH is a stable free radical with a characteristic absorption wavelength of $517 \mathrm{~nm}$. The pure PVA exhibited no antioxidant activity, as indicated by the similar peak strength in the control group. The UGS-PVA composite films exhibited excellent antioxidant activity as indicated by an evident reduction in peak strength. RSA increased from $20-85 \%$ as the UGS content increased. The color of the DPPH solution changed from purple to colorless, which was attributed to the reduction in DPPH by the antioxidants in UGS (Fig. S9). Usually, when fruits and vegetables suffered physical injuries, the injured areas would brown due to the polyphenol oxidation (Le Bourvellec et al. 2004). Hence, the antioxidant activity of UGS-PVA film further was demonstrated via attaching the film on a fresh apple. As showed in Fig. 5e, the peeled area attached with UGS15-PVA film had the slightest discoloration after the apple placed in the atmospheric environment for 3 hours. That was owed to the excellent antioxidant activity of the composite film, and the excellent adhesion (Fig. 4b) which could effectively isolate oxygen. Therefore, both above functions endowed the composite film a huge potential in food safety and packing.

The polyphenols with high UV absorption capacity (Fig. S10) endowed the UGS-PVA films with excellent UV-shielding. Compared with the pure PVA films, the UGS-PVA films showed superior UV-shielding performance with low UV transmittance (Fig. 5d). The positive synergistic effect of excellent antioxidant activity and UV-shielding performance provides such composite films great application potential for the repair of skin damage caused by UV illumination (Li et al. 2019; Wang et al. 2019). Moreover, the UGSPVA film possessed antimicrobial properties (Fig. 5f). After immersion, the films in milk and storage at room temperature for 21 days, as well as the milk containing the PVA film, was entirely covered by fungi. By contrast, the UGS ${ }_{15}$-PVA film inhibited fungal growth, and the milk contained only a small number of fungi. These observations are assumed to be related to the antimicrobial activity of grape polyphenols, which migrated from the composite film into the milk (Hassan et al. 2019; Katalinić et al. 2010). In addition, polyphenols had other functions, such as enhancing visual acuity and providing antiinflammatory and anti-cancer properties(Ju and Howard 2003; Udenigwe et al. 2008), thus expanding the potential applications of the UGS-PVA film.

\section{Conclusion}

In summary, a facile process via ultrasonication dissociation of GS and further compositing with PVA was developed to prepare a multifunctional UGS-PVA composite film. The efficient dissociation by 
ultrasonication resulted in a transparent and homogenously distributed UGS-PVA composite film. Similar to the pure PVA film, the UGS-PVA film retained its moisture-sensitive mechanical properties, particularly the significant improvement in breaking elongation at high MC. Notably, the retention of polyphenols in the UGS resulted in the multiple functions of the UGS-PVA films, such as $\mathrm{pH}$-sensitive color change, excellent antioxidant properties with an RSA of $85 \%$, excellent UV-shielding performance, and antimicrobial properties. These findings provide a new route for the resourceful utilization of waste GS. The UGS-PVA films exhibit significant potential for application in packaging, cosmetics, and healing materials.

\section{Declarations}

\section{Declaration of Competing Interest}

The authors declare no competing financial interest.

\section{Acknowledgements}

The Natural Science Foundation of Heilongjiang Province of China (YQ2019C003) and the National Training Program of Innovation and Entrepreneurship for Undergraduates of Northeast Forestry University (201910225180).

\section{References}

1. ASTM D3981-09a (2016), Standard Specification for Polyethylene Films Made from Medium-Density Polyethylene for General Use and Packaging Applications. ASTM International, West Conshohocken, PA.

2. Beres C, Costa GNS, Cabezudo I, da Silva-James NK, Teles ASC, Cruz APG, Mellinger-Silva C, Tonon RV, Cabral LMC, Freitas SP (2017) Towards integral utilization of grape pomace from winemaking process: A review. Waste Manage 68: 581-594.

3. Castillo-Muñoz N, Gómez-Alonso S, García-Romero E, Gómez MV, Velders AH, Hermosín-Gutiérrez I (2009) Flavonol 3-O-Glycosides Series of Vitis vinifera Cv. Petit Verdot Red Wine Grapes. J Agr Food Chem 57: 209-219.

4. Chen W, Yu H, Liu Y, Chen P, Zhang M, Hai Y (2011) Individualization of cellulose nanofibers from wood using high-intensity ultrasonication combined with chemical pretreatments. Carbohyd Polym 83: 1804-1811.

5. de Moura RS, Viana FSC, Souza MAV, Kovary K, Guedes DC, Oliveira EPB, Rubenich LMS, Carvalho LCRM, Oliveira RM, Tano T, Correia MLG (2002) Antihypertensive, vasodilator and antioxidant effects of a vinifera grape skin extract. J Pharm Pharmacol 54: 1515-1520. 
6. Einhorn-Stoll U, Kunzek H, Dongowski G (2007) Thermal analysis of chemically and mechanically modified pectins. Food Hydrocolloid 21: 1101-1112.

7. Esquivel-Alvarado D, Muñoz-Arrieta R, Alfaro-Viquez E, Madrigal-Carballo S, Krueger CG, Reed JD (2020) Composition of Anthocyanins and Proanthocyanidins in Three Tropical Vaccinium Species from Costa Rica. J Agr Food Chem 68: 2872-2879.

8. Fan QC, Jiang CJ, Wang WX, Bai LJ, Chen H, Yang HW, Wei DL, Yang LX (2020) Eco-friendly extraction of cellulose nancrystals from grape pomace and construction of self-healing nanocomposite hydrogels. Cellulose 27: 2541-2553.

9. Farooque S, Rose PM, Benohoud M, Blackburn RS, Rayner CM (2018) Enhancing the Potential Exploitation of Food Waste: Extraction, Purification, and Characterization of Renewable Specialty Chemicals from Blackcurrants (Ribes nigrum L.). J Agr Food Chem 66: 12265-12273.

10. Ferrari V, Taffarel SR, Espinosa-Fuentes E, Oliveira MLS, Saikia BK, Oliveira LFS (2019) Chemical evaluation of by-products of the grape industry as potential agricultural fertilizers. J Clean Prod 208: 297-306.

11. Han W, Ren J, Xuan H, Ge L (2018) Controllable degradation rates, antibacterial, free-standing and highly transparent films based on polylactic acid and chitosan. Colloid Surface A 541: 128-136.

12. Hassan YI, Kosir V, Yin X, Ross K, Diarra MS (2019) Grape Pomace as a Promising Antimicrobial Alternative in Feed: A Critical Review. J Agr Food Chem 67: 9705-9718.

13. Jin Q, Wang Z, Feng Y, Kim YT, Stewart AC, O'Keefe SF, Neilson AP, He Z, Huang H (2020) Grape pomace and its secondary waste management: Biochar production for a broad range of lead $(\mathrm{Pb})$ removal from water. Environ Res 186: 109442.

14. Ju ZY, Howard LR (2003) Effects of Solvent and Temperature on Pressurized Liquid Extraction of Anthocyanins and Total Phenolics from Dried Red Grape Skin. J Agr Food Chem 51: 5207-5213.

15. Kaboorani A, Riedl B, Blanchet P, Fellin M, Hosseinaei O, Wang S (2012) Nanocrystalline cellulose (NCC): A renewable nano-material for polyvinyl acetate (PVA) adhesive. Eur Polyme J 48: 1829-1837.

16. Katalinić V, Možina SS, Skroza D, Generalić I, Abramovič H, Miloš M, Ljubenkov I, Piskernik S, Pezo I, Terpinc P, Boban M (2010) Polyphenolic profile, antioxidant properties and antimicrobial activity of grape skin extracts of 14 Vitis vinifera varieties grown in Dalmatia (Croatia). Food Chem 119: 715723.

17. Kim JW, Park H, Lee G, Jeong YR, Hong SY, Keum K, Yoon J, Kim MS, Ha JS (2019) Paper-Like, Thin, Foldable, and Self-Healable Electronics Based on PVA/CNC Nanocomposite Film. Adv Funct Mater 29: 1905968.

18. Konidari MV, Papadokostaki KG, Sanopoulou M (2011) Moisture-induced effects on the tensile mechanical properties and glass-transition temperature of poly(vinyl alcohol) films. J Appl Polym Sci 120: 3381-3386.

19. Kozioł A, Cybulska J, Pieczywek PM, Zdunek A (2017) Changes of pectin nanostructure and cell wall stiffness induced in vitro by pectinase. Carbohyd Polym 161: 197-207. 
20. López de Lerma N, Moreno J, Peinado RA (2014) Determination of the Optimum Sun-Drying Time for Vitis vinifera L. cv. Tempranillo Grapes by E-nose Analysis and Characterization of Their Volatile Composition. Food Bioprocess Tech 7: 732-740.

21. Lavelli V, Sri Harsha PSC, Ferranti P, Scarafoni A, lametti S (2016) Grape skin phenolics as inhibitors of mammalian a-glucosidase and a-amylase - effect of food matrix and processing on efficacy. Food Funct 7: 1655-1663.

22. Le Bourvellec C, Le Quéré J, Sanoner P, Drilleau JF, Guyot S (2004) Inhibition of Apple Polyphenol Oxidase Activity by Procyanidins and Polyphenol Oxidation Products. J Agr Food Chem 52: 122-130.

23. Lecas M, Brillouet JM (1994) Cell wall composition of grape berry skins. Phytochemistry 35: 12411243.

24. Li L, Huang T, Lan C, Ding H, Yan C, Dou Y (2019) Protective effect of polysaccharide from Sophora japonica $L$. flower buds against UVB radiation in a human keratinocyte cell line (HaCaT cells). J Photoch Photobio B 191: 135-142.

25. Liu C, Shen W, L, B, Li T, Chang H, Cheng Y (2019a) Natural Polyphenols Augment Cytosolic Protein Delivery by a Functional Polymer. Chem Mater 31: 1956-1965.

26. Liu H, Xiang H, Li Z, Meng Q, Li P, Ma Y, Zhou H, Huang W (2020) Flexible and Degradable Multimodal Sensor Fabricated by Transferring Laser-Induced Porous Carbon on Starch Film. ACS Sustain Chem Eng 8: 527-533.

27. Liu J, Tan Y, Zhou H, Muriel Mundo JL, McClements DJ (2019b) Protection of anthocyanin-rich extract from $\mathrm{pH}$-induced color changes using water-in-oil-in-water emulsions. J Food Eng 254: 1-9.

28. Liu Y, Wang S, Lan W (2018) Fabrication of antibacterial chitosan-PVA blended film using electrospray technique for food packaging applications. Int J Biol Macromol 107: 848-854.

29. Mateus N, Silva AMS, Santos-Buelga C, Rivas-Gonzalo JC, de Freitas V (2002) Identification of Anthocyanin-Flavanol Pigments in Red Wines by NMR and Mass Spectrometry. J Agr Food Chem 50: 2110-2116.

30. Matharu AS, Houghton JA, Lucas-Torres C, Moreno A (2016) Acid-free microwave-assisted hydrothermal extraction of pectin and porous cellulose from mango peel waste - towards a zero waste mango biorefinery. Green Chem 18: 5280-5287.

31. Mendes JAS., Prozil S., Evtuguin DV, Lopes LPC (2013) Towards comprehensive utilization of winemaking residues: Characterization of grape skins from red grape pomaces of variety Touriga Nacional. Ind Crop Prod 43: 25-32.

32. Ortega-Regules A, Ros-García JM, Bautista-Ortín AB, López-Roca JM, Gómez-Plaza E (2008) Changes in skin cell wall composition during the maturation of four premium wine grape varieties. J.Sci Food Agr 88: 420-428.

33. Papadopoulou EL, Paul UC, Tran TN, Suarato G, Ceseracciu L, Marras S, d'Arcy R, Athanassiou A (2019) Sustainable Active Food Packaging from Poly(lactic acid) and Cocoa Bean Shells. ACS Appl Mater Inter 11: 31317-31327. 
34. Pinelo M, Arnous A, Meyer AS (2006) Upgrading of grape skins: Significance of plant cell-wall structural components and extraction techniques for phenol release. Trends Food Sci Tech 17: 579590.

35. Prozil SO, Evtuguin DV, Lopes LPC (2012) Chemical composition of grape stalks of Vitis vinifera L. from red grape pomaces. Ind Crop Prod 35: 178-184.

36. Quijada-Morín N, Hernández-Hierro JM, Rivas-Gonzalo JC, Escribano-Bailón MT (2015) Extractability of Low Molecular Mass Flavanols and Flavonols from Red Grape Skins. Relationship to Cell Wall Composition at Different Ripeness Stages. J Agr Food Chem 63: 7654-7662.

37. Serra AT, Matias AA, Nunes AVM, Leitão MC, Brito D, Bronze R, Silva S, Pires A, Crespo MT, San Romão MV, Duarte CM (2008) In vitro evaluation of olive- and grape-based natural extracts as potential preservatives for food. Innov. Food Sci Emerg 9: 311-319.

38. Šešlija S, Nešić A, Ružić J, Kalagasidis Krušić M, Veličković S, Avolio R, Santagata G, Malinconico M (2018) Edible blend films of pectin and poly(ethylene glycol): Preparation and physico-chemical evaluation. Food Hydrocolloid 77: 494-501.

39. Sigurdson GT, Tang P, Giusti MM (2017) Natural Colorants: Food Colorants from Natural Sources. Annu Rev Food Sci T 8: 261-280.

40. Štavíková L, Polovka M, Hohnová B, Karásek P, Roth M (2011) Antioxidant activity of grape skin aqueous extracts from pressurized hot water extraction combined with electron paramagnetic resonance spectroscopy. Talanta 85: 2233-2240.

41. Sun K, Li F, Li J, Li J, Zhang C, Chen S, Sun X, Cui J (2019) Optimisation of compatibility for improving elongation at break of chitosan/starch films. RSC Adv 9: 24451-24459.

42. Tu H, Zhu M, Duan B, Zhang L (2020) Recent Progress in High-Strength and Robust Regenerated Cellulose Materials. Adv Mater n/a: 2000682.

43. Udenigwe CC, Ramprasath VR, Aluko RE, Jones PJ (2008) Potential of resveratrol in anticancer and anti-inflammatory therapy. Nutr Rev 66: 445-454.

44. Wang H, Liu J, Pang D, Li T, Liu RH (2019) Mechanisms underlying the protective effects of blueberry extract against ultraviolet radiation in a skin cell co-culture system. J Funct Foods 52: 603-610.

45. Wang H, Qian J, Ding F (2018) Emerging Chitosan-Based Films for Food Packaging Applications. J Agr Food Chem 66: 395-413.

46. Wang X, Pang Z, Chen C, Xia Q, Zhou Y, Jing S, Wang R, Ray U, Gan W, Li C, Chen G, Foster B, Li T, Hu L (2020) All-Natural, Degradable, Rolled-Up Straws Based on Cellulose Micro- and Nano-Hybrid Fibers. Adv Funct Mater 30: 1910417.

47. Xu Y, Willis S, Jordan K, Sismour E (2018) Chitosan nanocomposite films incorporating cellulose nanocrystals and grape pomace extracts. Packag. Technol Sci 31: 631-638.

48. Xu ZY, Jiang XD, Zhou H, Li JY (2018) Preparation of magnetic hydrophobic polyvinyl alcohol (PVA)cellulose nanofiber (CNF) aerogels as effective oil absorbents. Cellulose 25: 1217-1227. 
49. Yang Y, Zhao Y, Liu J, Nie Z, Ma J, Hua M, Zhang Y, Cai X, He X (2020) Flexible and Transparent HighDielectric-Constant Polymer Films Based on Molecular Ferroelectric-Modified Poly(Vinyl Alcohol). ACS Mater Lett 2: 453-460.

50. Yu M, Zheng Y, Tian J (2020) Study on the biodegradability of modified starch/polylactic acid (PLA) composite materials. RSC Adv 10: 26298-26307.

51. Zhang Y, Pu C, Tang W, Wang S, Sun Q (2020) Effects of four polyphenols loading on the attributes of lipid bilayers. J Food Eng 282: 110008.

52. Zhu F, Du B, Zheng L, Li J (2015) Advance on the bioactivity and potential applications of dietary fibre from grape pomace. Food Chem 186: 207-212.

53. Zhu X, Liu B, Zheng S, Gao Y (2014) Quantitative and structure analysis of pectin in tobacco by $13 \mathrm{C}$ CP/MAS NMR spectroscopy. Anal Methods-UK 6: 6407-6413.

\section{Figures}

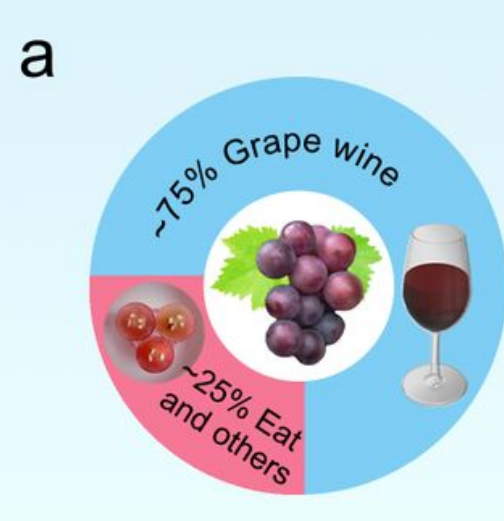

$>60$ Million Tons/Year b

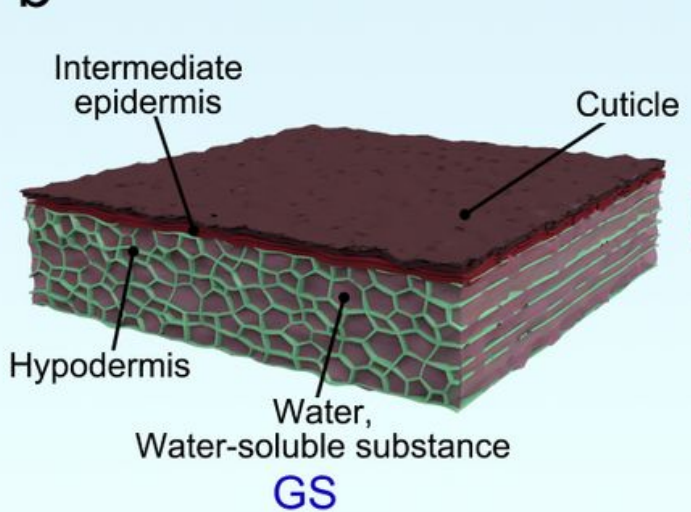

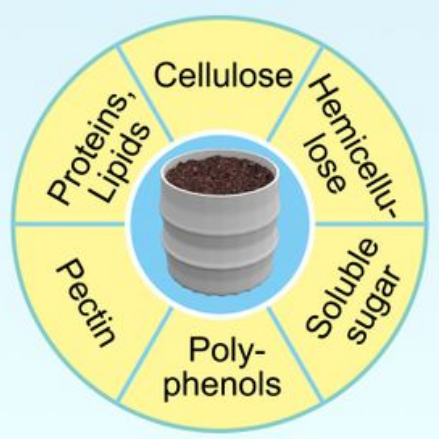

Components of GS

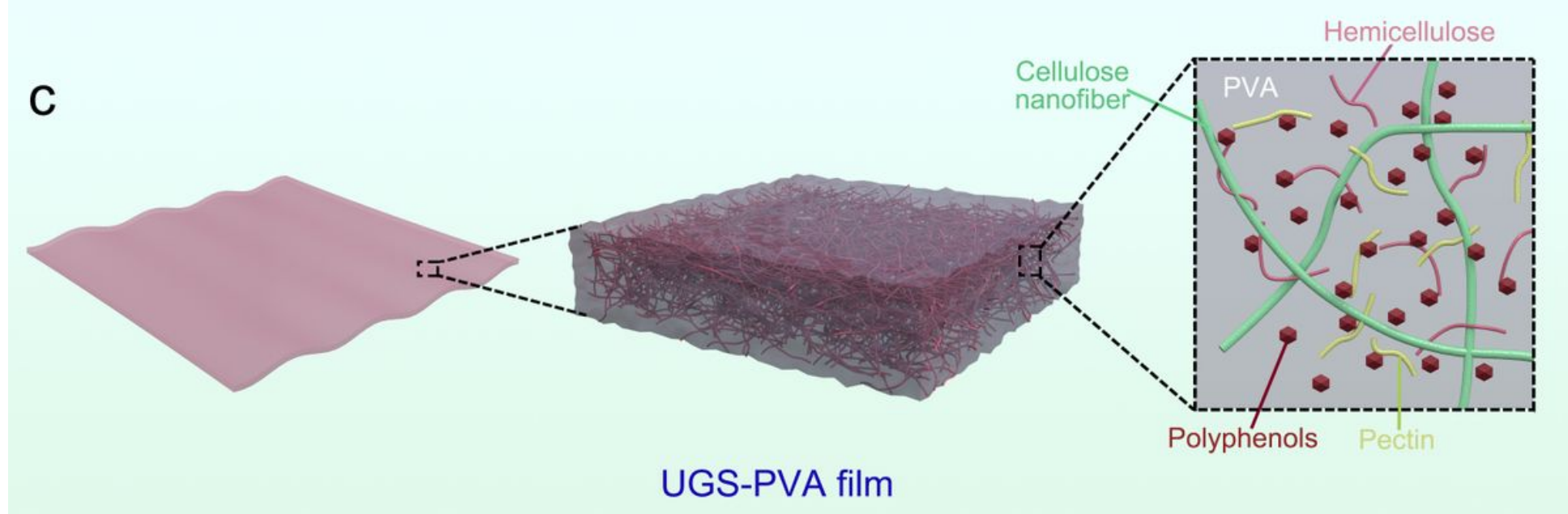

\section{Figure 1}

a Yield and utilization of grapes. b Structure and components of natural GS. c Structure and components of ultrasonicated UGS-PVA films. 


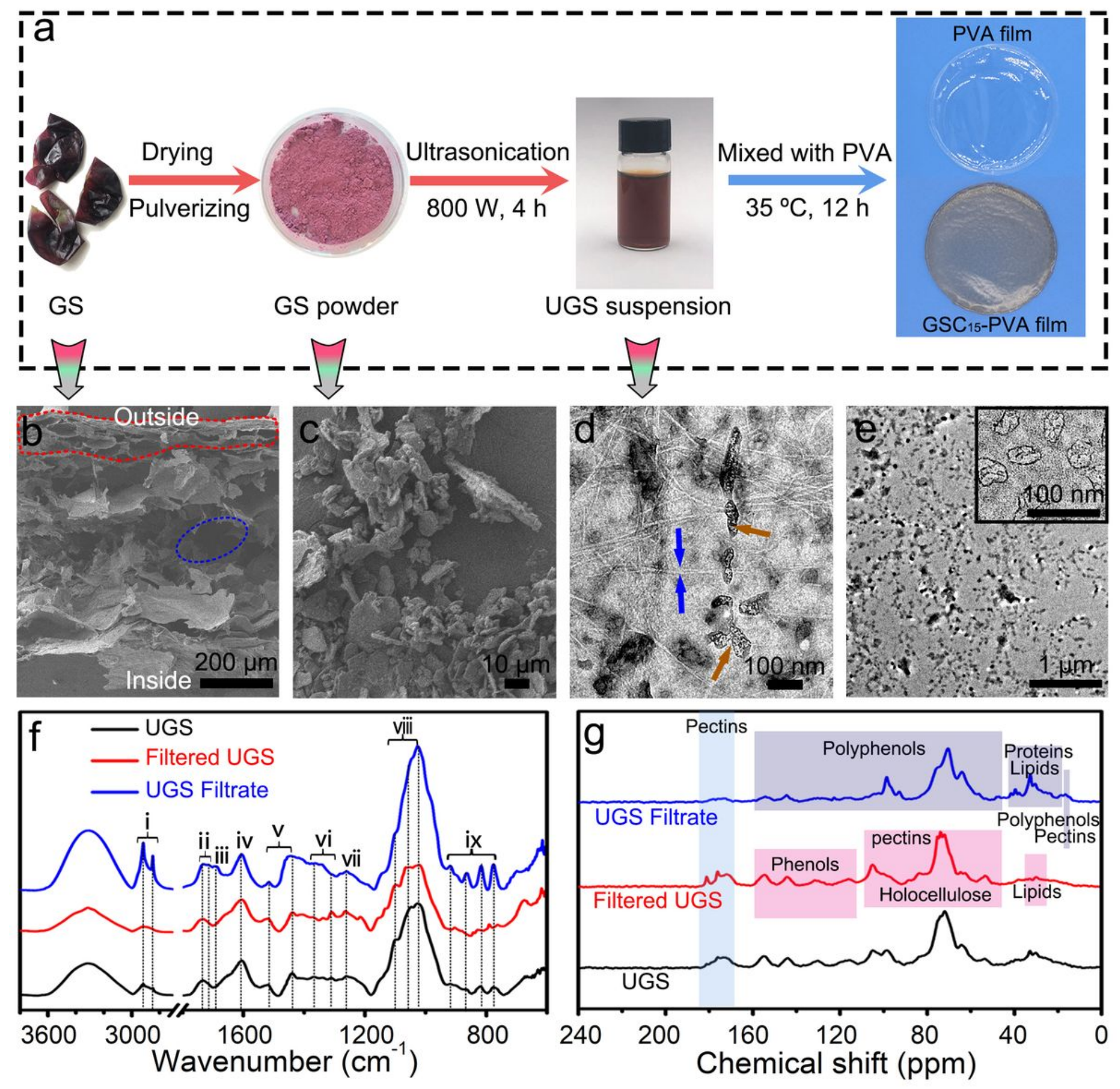

Figure 2

a Preparation of UGS and UGS-PVA composite films. b-c SEM images of the dried grape skin (GS) b and GS powder $c$. $d$ TEM image of the UGS suspension. e TEM images of the UGS suspension after filtering through a $450 \mathrm{~nm}$ ultrafiltration membrane at room temperature. $\mathrm{f}-\mathrm{g}$ FTIR absorption spectra $\mathrm{f}$ and $13 \mathrm{C}$ NMR spectra $g$ of the dried UGS, filtered UGS, and UGS filtrate. The red dashed area in $b$ is the cuticle and intermediate epidermis, and the blue ovals in b indicate the vessel structure in thehypodermis of the GS. 
The blue arrows in $d$ are the CNF, and the brown arrows in $d$ are the aggregates, likely consisting of polyphenols, pectin, and soluble sugar. The inset in e shows the high-magnification TEM image.

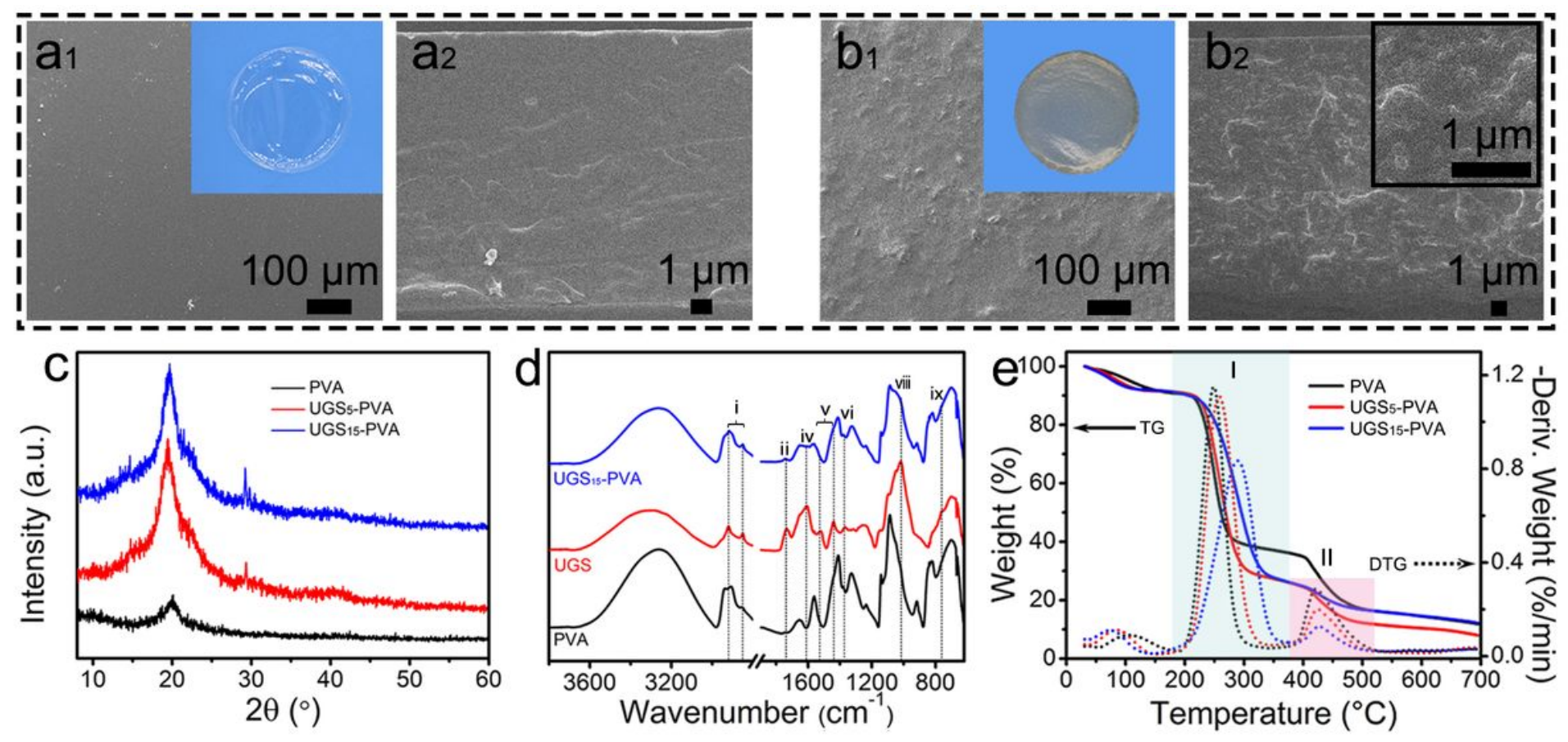

\section{Figure 3}

$a-b$ SEM images of a PVA and b UGS15-PVA films: $a 1$ and $b 1$ are the cross-sections, $a 2$ and $b 2$ are the surfaces. c XRD patterns of pure PVA, UGS5-PVA, and UGS15-PVA films. d FTIR spectra of PVA, UGS, and UGS15-PVA films. The serial numbers of the peaks were consistent with those in Fig. 2f. e TG and DTG curves with temperatures ranging from room temperature to $700^{\circ} \mathrm{C}$. The inserts in $\mathrm{a} 1 \mathrm{and} \mathrm{b} 1$ were the photographs of the films. 

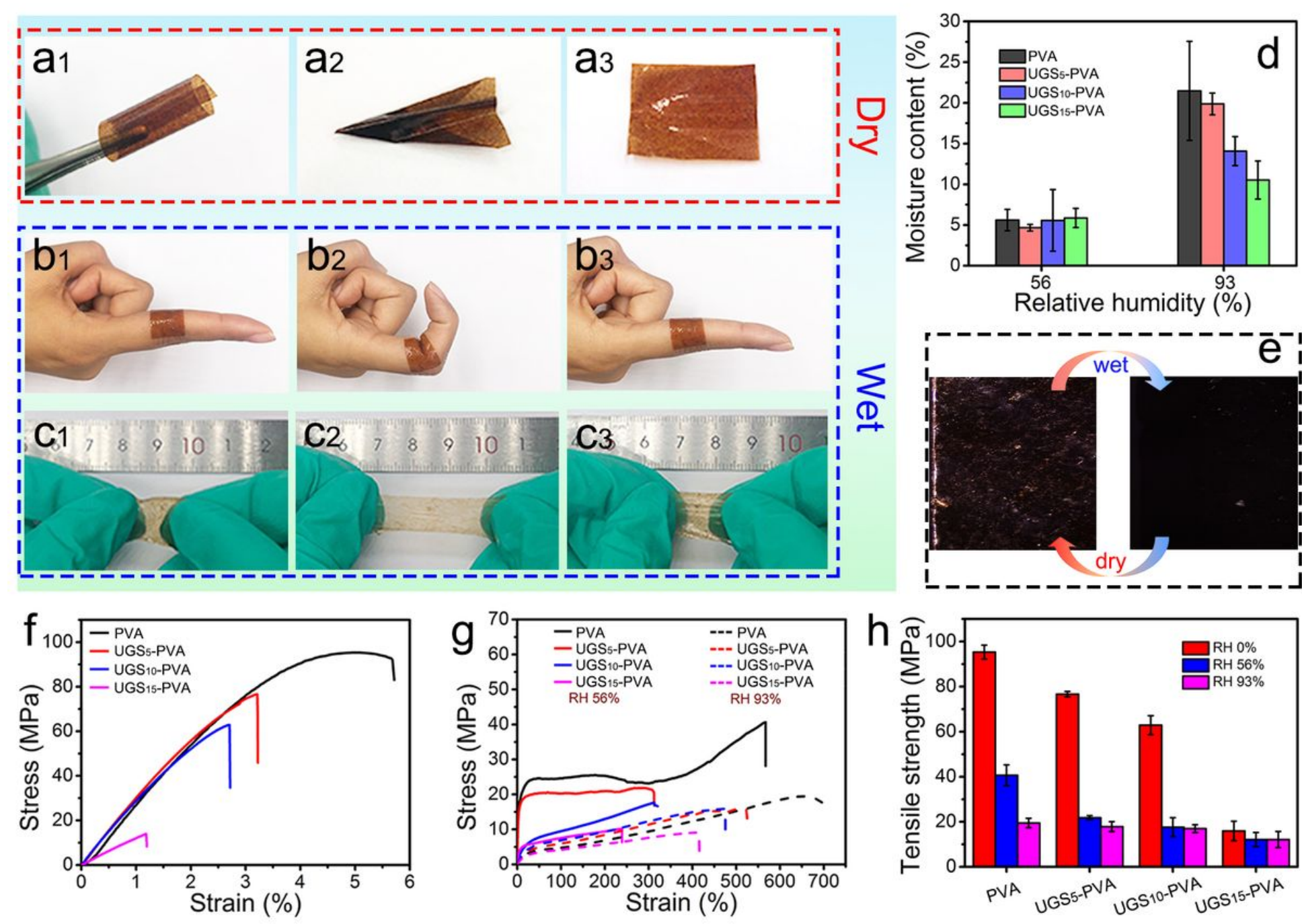

Relative humidity $(\%)$
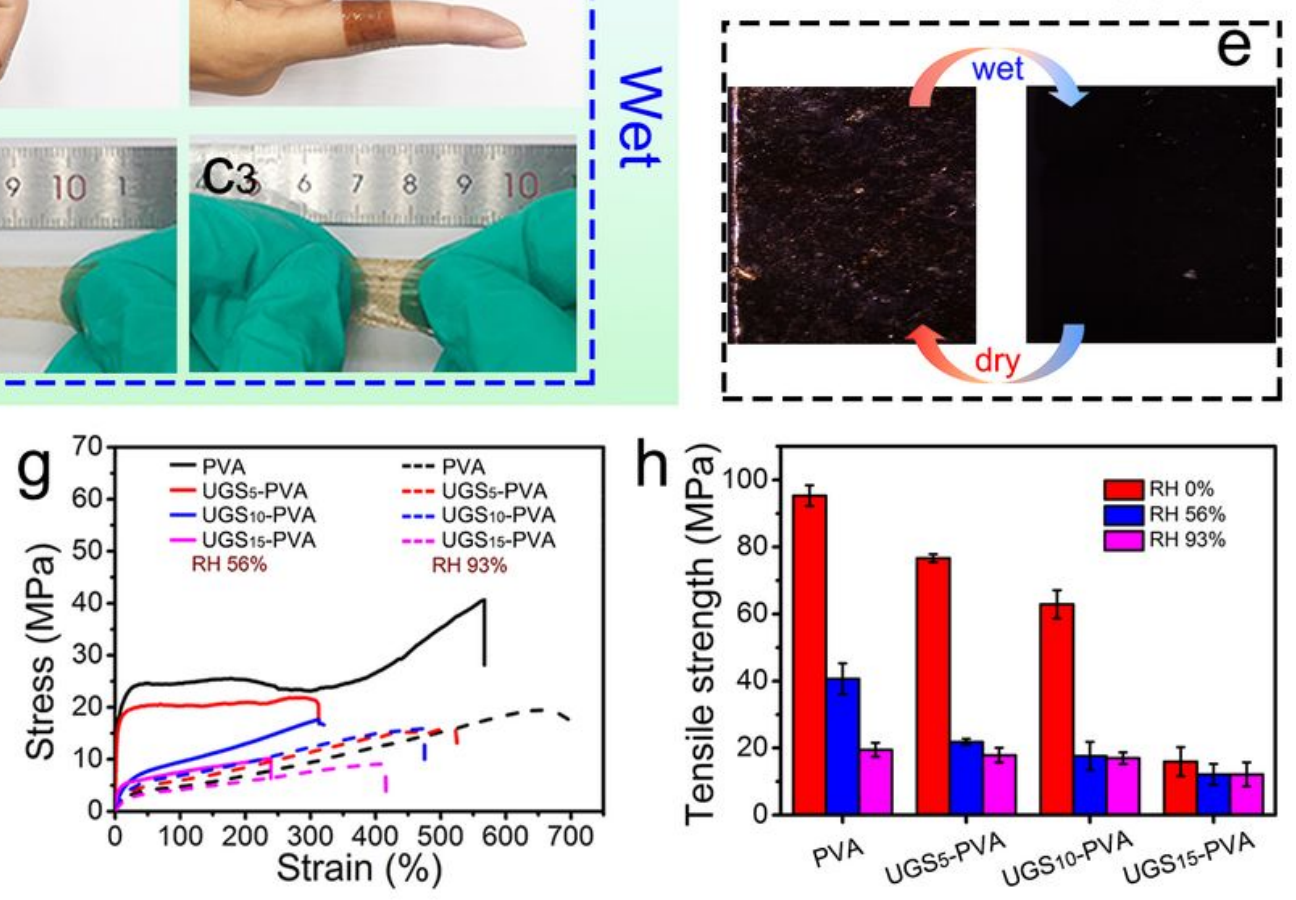

\section{Figure 4}

Images of the flexible UGS5-PVA film under different conditions: a the dry film is folded and unfolded without damage; $b$ the film wetted in deionized water for 5 minis attached to the finger joint and is deformed when the finger is bent; c stretching and retraction of the wet film. $d$ Moisture content of the films at relative humidity $(\mathrm{RH})$ levels of $56 \%$ and $93 \%$. e Polarizing microscope images of the UGS15PVA film in dry and wet states. $f-g$ Stress-strain curves of the films after equilibrating $f$ at $\mathrm{RH}$ of $0 \%, \mathrm{~g}$ at $\mathrm{RH}$ of $56 \%$ and $93 \%$. h Tensile strength of the films after equilibrating at different $\mathrm{RH}$ levels. 

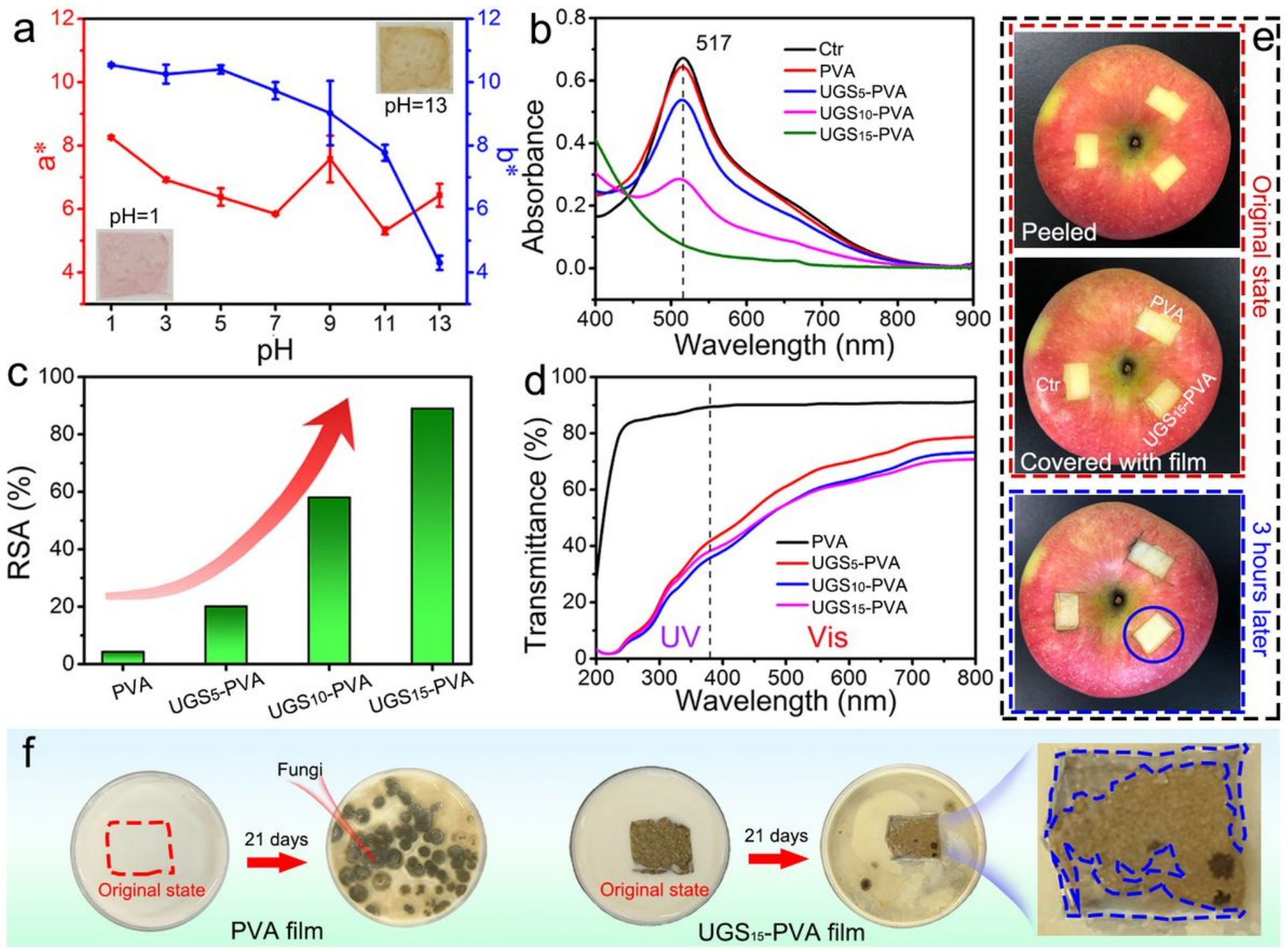

\section{Figure 5}

a pH-responsiveness of the UGS15-PVA film: change in color of the film at different $\mathrm{pH}$ values ( $\mathrm{a}^{\star}$ is redgreen, $b$ * is yellow-blue). b-c Antioxidant activity of the migrated components of different UGS-PVA films: $b$ Decolorization of DPPH radicals in methanol attributed to the reaction with UGS-PVA film solutions (characteristic absorption wavelength of DPPH, $517 \mathrm{~nm}$ ), c Radical scavenging activity (RSA) of different UGS-PVA films. d Optical transmittance of the films. e Demonstrating the antioxidant properties of the film on a fresh apple. After placed in atmospheric environment for 3 hours, the films were removed, and the blue area with the UGS15-PVA film exhibited the slightest discoloration. $f$ Fungal growth in milk in the presence of PVA or UGS15-PVA films after 21 days in the laboratory. The blue dashed area in the magnified image is transparent owing to the dissolution and release of the colored polyphenols.

\section{Supplementary Files}

This is a list of supplementary files associated with this preprint. Click to download.

- 2SupplementaryInformation20210120.doc 